\title{
Norms and values in sociohydrological models
}

\author{
Mahendran Roobavannan ${ }^{1}$, Tim H. M. van Emmerik ${ }^{2}$, Yasmina Elshafei ${ }^{3}$, Jaya Kandasamy ${ }^{1}$, \\ Matthew R. Sanderson ${ }^{4}$, Saravanamuthu Vigneswaran ${ }^{1}$, Saket Pande ${ }^{2}$, and Murugesu Sivapalan ${ }^{5,6}$ \\ ${ }^{1}$ School of Civil and Environmental Engineering, University of Technology Sydney, Sydney, NSW, Australia \\ ${ }^{2}$ Department of Water Management, Delft University of Technology, Delft, the Netherlands \\ ${ }^{3}$ School of Earth \& Environment, University of Western Australia, Crawley, WA 6009, Australia \\ ${ }^{4}$ Department of Sociology, Kansas State University, Manhattan, KS 66506, USA \\ ${ }^{5}$ Department of Civil and Environmental Engineering, University of Illinois at Urbana-Champaign, Urbana, IL 61801, USA \\ ${ }^{6}$ Department of Geography and Geographic Information Science, University of Illinois at Urbana-Champaign, \\ Champaign, IL 61820, USA
}

Correspondence: Jaya Kandasamy (jaya.kandasamy@uts.edu.au)

Received: 19 July 2017 - Discussion started: 26 July 2017

Revised: 14 November 2017 - Accepted: 1 January 2018 - Published: 23 February 2018

\begin{abstract}
Sustainable water resources management relies on understanding how societies and water systems coevolve. Many place-based sociohydrology (SH) modeling studies use proxies, such as environmental degradation, to capture key elements of the social component of system dynamics. Parameters of assumed relationships between environmental degradation and the human response to it are usually obtained through calibration. Since these relationships are not yet underpinned by social-science theories, confidence in the predictive power of such place-based sociohydrologic models remains low. The generalizability of SH models therefore requires major advances in incorporating more realistic relationships, underpinned by appropriate hydrological and social-science data and theories. The latter is a critical input, since human culture - especially values and norms arising from it - influences behavior and the consequences of behaviors. This paper reviews a key social-science theory that links cultural factors to environmental decision-making, assesses how to better incorporate social-science insights to enhance SH models, and raises important questions to be addressed in moving forward. This is done in the context of recent progress in sociohydrological studies and the gaps that remain to be filled. The paper concludes with a discussion of challenges and opportunities in terms of generalization of SH models and the use of available data to allow future prediction and model transfer to ungauged basins.
\end{abstract}

\section{Introduction}

The concept of sustainable development has received much attention among researchers, policy makers and stakeholders. Water is at the core of many of the sustainability challenges that human societies face (Bai et al., 2016; Falkenmark and Rockström, 2004; Rijsberman, 2006). Sustainable water resource management is key to production of food and energy to satisfy human needs, including poverty alleviation and human health. As indiscriminate development threatens critical ecosystem services and biodiversity, the need to account for the environment has emerged as an important consideration in sustainable water management (Millennium Ecosystem Assessment, 2005). Enabling society to address sustainability challenges, and develop appropriate solutions, requires an ability to provide reliable predictions of changes to freshwater resources and their distribution, circulation, and quality under natural and human-induced changes from local to global scales, including changes that are part of water management (Srinivasan et al., 2017).

We cannot understand, let alone make future predictions of, water resource system dynamics without understanding how the issues of economic gain, environmental degradation, and social inequities play out in society, and how social perceptions of these issues impact management decisions relating to water consumption, water allocation and pricing, human settlements, infrastructure development, and environmental protection (Blair and Buytaert, 2016; Srinivasan et 
al., 2016). Such understanding will remain incomplete until we fully grapple with issues arising from human culture, including how components of culture, i.e., values, beliefs, and norms related to water uses, livelihood, and the environment (Sivapalan and Blöschl, 2015). It is increasingly recognized that cultural factors are likely to influence changes in water management decisions and outcomes (Caldas et al., 2015), raising questions about what have become "conventional" assumptions about humans as rational utility maximizers who make decisions based upon complete information. Although economic models of altruism and impure altruism (i.e., "warm glow" effect: caring about others or the next generation not just out of altruism but because they get pleasure out of it themselves) have been successful in predicting the effect of prevailing values and norms on human behavior and actions (Andreoni, 1989; Banerjee and Newman, 1993), they remain limited in accounting for the consequences of the human actions on societal values and norms in return.

The interdisciplinary field of sociohydrology was launched with the aim of studying the dynamic, two-way feedbacks between water and people in coupled humanwater systems. In particular, sociohydrology (SH) seeks to understand and interpret patterns and phenomena that emerge from two-way feedbacks in coupled human-water systems as a consequence of water management decisions and actions. Indeed, the subject matter of sociohydrology includes the many diverse phenomena that emerge from these two-way feedbacks and manifest as puzzles and paradoxes, exhibiting differences but also similarities between places, and reflecting distinct hydroclimatic, eco-environmental, and socioeconomic backgrounds (Sivapalan et al., 2014). Examples include the agrarian crisis in booming emerging economies such as India (Pande and Savenije, 2016), increasing levee heights in urban environments in spite of increased flood risk (Di Baldassarre et al., 2013) and the peaking in water resource availability in agricultural basins as they undergo development (Kandasamy et al., 2014; Liu et al., 2014).

Several place-based sociohydrology studies in basins dominated by agricultural development, such as the Tarim (China, Liu et al., 2014), Murrumbidgee (Australia, Elshafei et al., 2014; van Emmerik et al., 2014), and Toolibin Lake (Australia, Elshafei et al., 2015) basins, have highlighted a shift in water use behavior from an initial focus on agricultural production to an increasing emphasis on environmental conservation, a shift that has been called the pendulum swing (Kandasamy et al., 2014). Similarly Chen et al. (2016) showed a shift in water management from flood mitigation to wetland protection at Kissimmee river, USA. Sociohydrology models developed to reproduce these observed dynamics attributed the shift to changing human values and norms, which were tracked indirectly through proxies (e.g., environmental degradation). For example, van Emmerik et al. (2014) modeled the human decision to allocate more or less wa- ter to agriculture or to the environment on the strength of a dynamic "social" state variable called environmental awareness, which reflected societal perceptions of the environmental degradation within the prevailing value systems or culture (see also Di Baldassarre et al., 2013, for awareness of floods in the context of coupled human-flood systems, and Garcia et al., 2016, for awareness of shortage for town water supply in the context of coupled human-town water supply systems). In the sociohydrological model of van Emmerik et al. (2014) the human response to changing environmental awareness is captured through an appropriate constitutive relationship, chosen in a somewhat intuitive way. Hence, the parameters governing the constitutive relationship could only be obtained through calibration of the overall model and would always be challenged unless they are verified to be right for the right reasons. Prediction-wise, both in time and space, confidence in such place-based models will be low so long as the constitutive relationship cannot be independently validated or theoretically justified.

Going forward, there is a need to generalize SH models both for predicting future sociohydrological outcomes in one location and/or to apply them at other locations. Case studies have demonstrated an inherently dynamic quality to changing values and norms in relation to water use or environmental behavior, but how to measure or "value" values and norms directly and independently of models remains as yet unresolved. Even if they can be measured in specific places, we need a broad theoretical framework that encapsulates the many physical and social controls that govern changing values and norms in order to synthesize data or measurements from many places across the globe and develop broad generalizations. These remain major challenges to the progress of sociohydrology as the science underpinning sustainable water management (Pande and Sivapalan, 2016) and thus provide the motivation for this paper. Our aim is to position the progress made by $\mathrm{SH}$ models to date towards incorporating changing values and norms in the context of extant socialscience theories, and in doing so, to articulate possible ways forward to make major advances in the future.

This paper begins with a review of recent place-based, sociohydrological modeling studies (van Emmerik et al., 2014; Elshafei et al., 2014, 2015; Chen et al., 2016; Roobavannan et al., 2017) that have incorporated changing values and norms by connecting them to measures of the states of basin economy and/or environmental health via assumed functional relationships. Next, we draw connections between extant social theory and recent SH studies that indicate how values and norms influence social behavior towards the environment. The paper then outlines challenges and opportunities for generalizing SH models, especially in respect of changing values and norms, so that more reliable predictions can be made across time and space. This includes a recalibration exercise to demonstrate the value of new kinds of social data. This also includes exciting new avenues such as virtual social experiments or data mined from novel sources such as social 
surveys and media. It concludes with the possibility of generalizing relationships between changing values and norms and human behavior in respect of the environment, benefiting from more place-based studies. In this way, it underscores the need for more comparative analyses across many such case studies so that generalized relationships can be synthesized that are transferrable to ungauged locations.

\section{Values and norms in sociohydrological models}

Following Wescoat (2013), the sociohydrology literature has tended to define values and norms as the overarching goals of individuals and of whole societies in respect of water use, conservation, and sustainability. Prior research in SH has allowed values and norms to undergo dynamic changes. Sivapalan et al. (2014) proposed a sociohydrological framework which uses values and norms as drivers of the decisionmaking that shapes society's goals and actions, and are in turn shaped by the outcomes for human well-being that result from past human decisions (Fig. 1). In this way, values and norms are seen as endogenous to coupled human-water systems, coevolving with the changing dynamics of water resource systems (Norton et al., 1998; Sivapalan and Blöschl, 2015). So far in SH research, values, beliefs, and norms have been lumped together and represented by proxy variables. Next, we illustrate this through several examples.

\subsection{Environmental awareness}

Van Emmerik et al. (2014) developed a SH model of the Murrumbidgee river basin (MRB) in eastern Australia to explain an observed "pendulum swing", i.e., a shift in water management focus away from economic development and towards ecosystem health. This shift was hypothesized to be the outcome of changes in values and norms in the community in respect of economic well-being and ecosystem health. In the model, the dynamics of changing values and norms were represented by environmental awareness, a proxy state variable that reflected adverse changes to ecosystem health. A crucial aspect has been the inclusion of a submodel to quantify environmental health. It was assumed that environmental degradation occurred when too much water was extracted for agricultural activities aimed at advancing the economic well-being of the community. As a result, less water reached downstream wetlands. When wetland storage became lower than a specified threshold, ecosystem health suffered noticeably to be felt in the community, which was then reflected in the environmental awareness. Enhanced environmental awareness then triggered human action, in the form of reductions in water allocation to agriculture, leading to reductions in irrigated area and increased water allocation to the environment. The situation would reverse itself upon a return of increased downstream environmental flows, restoration of wetland storage, and improvement to ecosystem health.

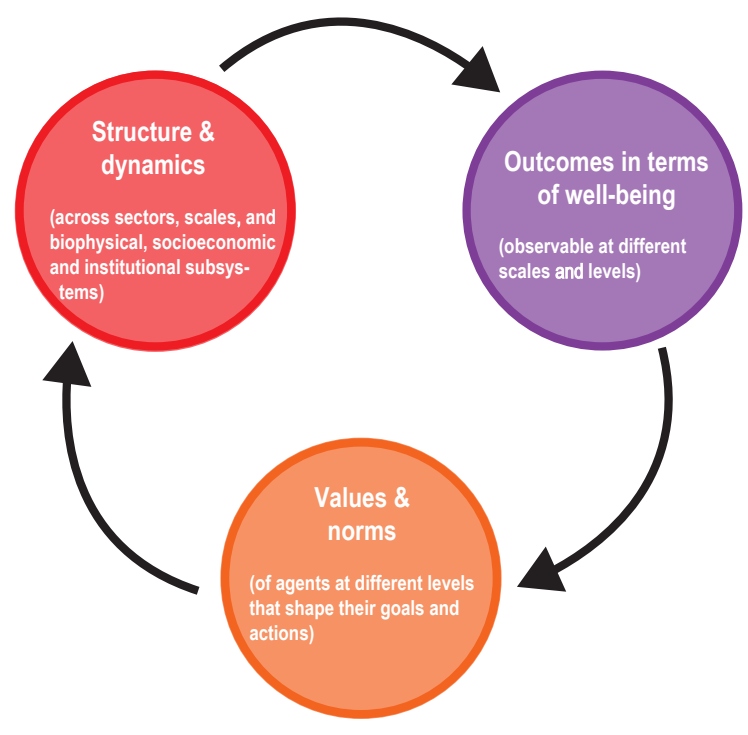

Figure 1. Framework proposed by Sivapalan et al. (2014). Sociohydrology models use proxies for environment degradation and for economic well-being.

The representation of environmental awareness in van Emmerik et al. (2014), although simple, represents one of the first attempts to study the intuitive relationship between values and norms about perceived threats to ecosystem health and changes to water management actions. Van Emmerik et al. (2014) was able to model the four eras described by Kandasamy et al. (2014), from an exclusive focus on agriculture to environmental restoration. Note that other effects or characteristics of environmental degradation, such as changing water tables or salinization of the soil, were not taken into account. Furthermore, regional or national economy and policies and changing perceptions are not taken into account in the formulation of environmental awareness. Finally, the functional form of the equation was calibrated using data on population, total irrigated area, and agricultural water utilization.

\subsection{Community sensitivity}

Elshafei et al. (2014) expanded further on the intuitive causality between changes to community values and norms in respect of ecosystem health, regional economy, and consequent water management actions by humans. They elaborated on how agricentric values conflicted with environmental values and influenced water use behavior and proposed a framework that modeled the competition between economic development and environmental awareness using "community sensitivity", a new social state variable. They presented a feedback formulation where water use behavior is influenced by changing values and norms relating to the environment and economic well-being, as reflected in the community sensitivity. For the first time the authors brought 
in broader (e.g., regional) climatic, political, and socioeconomic contextual variables that may influence local values and norms in respect of water use, e.g., rapidly diversifying economic growth. Elshafei et al. (2015) explicitly demonstrated that environmental degradation impacted community sensitivity and consequently water use behaviors. The foundation of their proposed framework was driven by the hypothesis that the coupled system dynamics are driven by the competition between a positive feedback loop (economicpopulation loop) and a negative feedback loop (community sensitivity loop).

Similarly, the community sensitivity concept was used to explain the shift in values and norms as well as the shift in management emphasis from flood mitigation to environment protection in Kissimmee river, Florida, USA (Chen et al., 2016). They used wetland storage and flood intensity as proxies to measure changing value system. Their study showed that the value system was affected by the relative size of population in upstream and downstream portions of the catchment.

\section{Economic diversification and institutions}

Roobavannan et al. (2017) presented a rigorous validation of the community sensitivity concept of Elshafei et al. (2014) and further extended it to account for the relative dependence of the basin economy on agriculture. Roobavannan et al. (2017) assumed that the tradeoff between economic well-being and environmental health at the community level also depends on contextual factors such as economic diversification. In this way the resulting SH model was able to explain the importance of economic diversification and sectoral transformation for the community sensitivity that then impacted human water management actions.

Roobavannan et al. (2017) also introduced a fish species richness (FSR) index (Yoshikawa et al., 2014) as a separate proxy for ecosystem health. They also used time series of economic development (measured by total irrigated area and irrigation water utilization) and diverse proxies for technology (i.e., patents) and water use behavior (e.g., environmental behavior based on fish species richness index) to validate the dynamic changes to community sensitivity.

Community sensitivity and environmental awareness are different in the way they are defined, yet both intend to capture the same concept of changing values and norms for use in sociohydrological models. Environmental awareness accounts for society's perception of environment degradation while community sensitivity accounts for the balance of perception of environment degradation and economy growth of a region. Community sensitivity is a more complex assessment variable than environmental awareness. Both are modeled as memory variables. But while in the case of the latter the timescale of the memory of past environmental disaster is kept constant, in the case of the former (i.e. community sen- sitivity) the timescale is dynamic and depends on community norms in the context of its water environment.

\section{Values, beliefs, and norms as dynamic variables}

So far in SH modeling research, aspects of human culture that drive human behavior in respect of water management i.e., values and norms - have been treated in a lumped way, represented by proxies, in a "black-box" manner. Moving SH forward requires the black box of culture to be opened by questioning the assumptions behind, and more clearly measuring and modeling, cultural factors. For example, if values are conceptualized as overarching goals of society (Wescoat, 2013), are they individual goals or collective goals associated with the emergent structure of a coupled human-water system, or both? Similarly, how malleable are values and norms as aspects of a coupled human-water system? Moreover, under what conditions should values and norms be expected to change, or remain stable? For that matter, what are the mechanisms through which values and norms might change, and the human behaviors and actions that result from them?

The ingredients for understanding the role of changing values and norms in coupled human-water systems can be summarized as follows: (a) forward loop - theories of how individual values influence individual norms and behavior regarding water use; (b) backward loop - theories of why and how collective behavior can engender change in individual norms regarding the use of water for agriculture or the environment; (c) the role of institutions in enabling changes in water policy that reflect collective behavior towards the water environment; (d) data that can provide information on proxy variables including environment-related behavior and patterns; and (e) models that use proxy data to conceptualize processes (a)-(c) in interpreting related patterns. Future work in SH will necessarily grapple with these types of questions that further elucidate the role of values and norms in coupled human-water systems.

\subsection{Values, beliefs, and norms: VBN theory}

One line of conceptualization seems particularly promising for advancing sociohydrological research. The valuesbeliefs-norms (VBN) theoretical framework (Stern et al., 1999; Ives and Kendal, 2014) is grounded firmly in socialpsychological theory and has been empirically tested as a framework for understanding how cultural factors (i.e., values, beliefs, and norms) shape environmental decisionmaking, and water use behavior in particular, in a wide array of contexts. Figure 2 presents a stylized version of a VBN model linking values, beliefs, norms, and behaviors.

In the social sciences, "values" can have various meanings and definitions (Dietz, 2015). The social-science literature on values is voluminous, but there is a large strand of research that employs the meaning of values from Schwartz et 


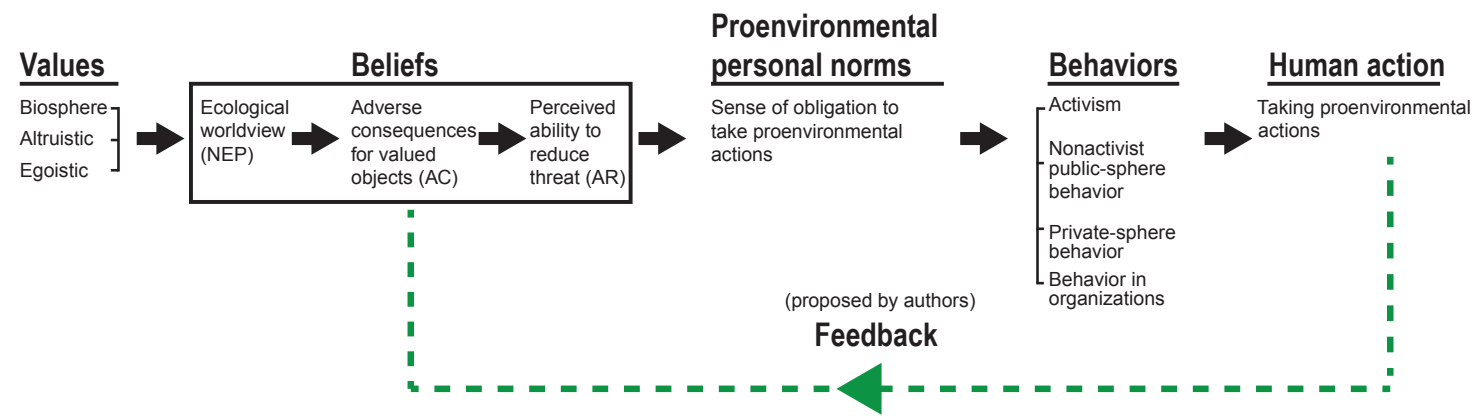

Figure 2. Value-belief-norm (VBN) theory. Adapted from Ives and Kendal (2014) and Stern (2000). The feedback (green arrow) from communal behavior to individual beliefs is introduced here by the authors to recognize that it has indeed been included in recent SH studies in preliminary ways (van Emmerik et al., 2014, Elshafei et al., 2014, and Roobavaanan et al., 2017) and needs to be formalized in future studies.

al. (2001, p. 521), which defined values as "desirable, transsituational goals, varying in importance that serve as guiding principles in people's lives". Values in this sense are different from beliefs and norms. Beliefs are ideas about what is true (or not); beliefs can be held regardless of empirical evidence. Norms are rules, written and formal or unwritten and informal, that prescribe behaviors. Norms specify how people should or should not act. Values - as guiding principles - motivate beliefs and norms and influence whether people accept particular beliefs and norms. In this framework, behaviors are motivated by proximate norms or obligations to act. Norms themselves are shaped or activated by beliefs, including a person's awareness of the consequences of their actions, how a person ascribes responsibility for their actions, etc. More generally, norms are shaped by a person's ecological world view, or how a person views humans visà-vis the natural environment (i.e., are humans a part of the natural environment, or apart from the natural environment). Ultimately, the VBN framework posits values - deeply held, guiding principles about right and wrong - as the basis of water use behavior in the context of sociohydrology. Values are often assumed to be unchanging, relatively stable, and generally unquestioned principles that motivate water use behavior and water policy actions indirectly through beliefs and norms.

The VBN framework is capable of being incorporated into $\mathrm{SH}$ models for the purposes of modeling dynamic feedbacks within the human component of the system or between the human and environmental components of the system (Caldas et al., 2015). Incorporating VBN into SH models requires the questions raised above to be addressed in greater detail, among others, but especially the question of where the feedbacks among values, beliefs, norms, and behavior occur in the process of management and the competitive use of water resources.

To illustrate how values, beliefs, and norms influence behavior (Fig. 2), consider a simplified example of a farmer of English descent in the MRB who migrated into the basin in the early 1900s and farmed rice. The behavior of this farmer towards wetlands is influenced by how the farmer and the farming community believe their water use affects what they hold dear or value. Implicitly, this means that their behavior towards the environment depends on how they value water, or what they believe the water should be used for. These are questions of values, and values help navigate decisions that must be made about trade-offs between differently valued end goals or uses. Here, one key trade-off is between water for agricultural production (i.e., to support the viability of the farm operation and farmer's livelihood) and water for the environment (i.e., to support environmental flows, biodiversity, and ecosystem services). Humans can hold multiple values and place different "weights" or emphases on each of the values that affect a particular decision with regards to water use. The farmer may, for example, make a water use decision by drawing on a combination of self-interest or egoistic values (e.g., using water to support the economic well-being of their family, household, and farm), humanist-altruistic values (e.g., conserving water to preserve the long-term viability of the rural community), and biospheric-altruistic values (e.g., conserving water to preserve wildlife habitat and ecosystem services). A first step toward modeling this type of VBN process could be to assign weights for each value, allowing behaviors to change in correspondence to the weights that each value type exercises over time. Scaling up from the individual level, value types can be identified from prevailing complexes of VBN processes in a basin so that SH dynamics in a basin are outcomes of generalized behaviors emerging from a distribution of basin residents laden with different value types and complexes. From this perspective, VBN elements at an aggregate level in a basin can become dynamic. For example, degrading ecosystem functioning, such as the drying of wetlands, can bring more uncertainty and risk over time to the things the farmer values (i.e., income, family, farming, community, the environment, etc.) and/or alter the farmer's beliefs (i.e., world view, awareness of adverse consequences, or perceived ability to reduce threats to things 
of value), shifting their behavior away from a more egoistic, or agricentric, orientation and towards wetland conservation and restoration. This is a very simplified example of a complex set of processes operating on multiple scales, but it illustrates how values, beliefs, norms, and behavior might be seen to coevolve and change through feedbacks in a coupled SH system.

Important gaps remain in how to identify the requisite components of VBN processes through measurement, how to scale up these processes from the individual level, and how to model feedbacks. However, as mentioned before, there has already been progress in this direction in the $\mathrm{SH}$ literature.

\subsection{Validation of modeled changing values and norms}

Place-based SH models have relied on proxy measures such as environmental degradation to capture changing values, beliefs, norms, and behaviors (Fig. 2), and their parameters were obtained by calibration. Despite the advantages of this approach, confidence in these models remains low, as the models are difficult to independently validate. To address the validation challenges faced to date in model-based sociohydrology case studies, Elshafei et al. (2015) proposed that sociocentric approaches (such as newspaper content analysis) be employed to assess evolving community sentiment over long time periods.

Along these lines, Xiong et al. (2016) and Wei et al. (2017) recently analyzed the content of newspaper articles to measure and quantify the evolution of societal values and norms in relation to water management issues in China and Australia. The results of Wei et al. (2017) are especially informative to the growing body of sociohydrology literature focused on Australian study sites, in particular the Murray-Darling Basin (MDB). Their findings support the hypothesis that societal values shifted from an anthropocentric to an envirocentric focus over time.

The work of Wei et al. (2017) thus signals an important step forward for the sociohydrology research community, as its results demonstrate how an autonomous sociocentric analysis method may be employed to provide independent validation for conceptual theories and coupled modeling approaches carried out within the same broad geographical region. This more complete analysis of societal values and norms now enables us to go back and compare the results of this independent study against the predictions made by previous SH models. More specifically, Wei et al.'s (2017) results corroborate Kandasamy et al.'s (2014) proposed pendulum swing in societal sentiment in the Murrumbidgee basin over a century-long timescale. As can be seen in Fig. 3, observed (Fig. 3a, Kandasamy et al., 2014) and modeled (Fig. 3b, van Emmerik et al., 2014) time series of economic development (proxied by total irrigated area and irrigation water utilization) correspond with the evolution of societal sentiment shown in the bottom panel of Wei et al.'s (2017) results (Fig. 3c). Moreover, the narrative for each of the three phases described in Wei et al. (2017) repeats the timing and spirit of the phases depicted in Kandasamy et al. (2014), van Emmerik et al. (2014), and Elshafei et al. (2014, 2015) (Fig. 3).

Another important implication of Wei et al.'s (2017) results is that they provide strong support for theories underpinning the use of the composite "community sensitivity" variables. Figure $4 \mathrm{a}$ and $\mathrm{b}$ illustrates that when societal values are initially focused on economic development the change in the community sensitivity variable $(\mathrm{d} V / \mathrm{d} t)$ trends negatively (i.e., society is predisposed towards anthropocentric behaviors), whereas as societal values evolve towards environmental sustainability the change in community sensitivity variable trends positively (indicating a behavioral tendency towards conservation). Wei et al.'s (2017) findings thus provide strong validation for the nonlinear dynamics observed in previously published coupled SH models that adopted alternate proxies for modeling the change in societal values and norms in relation to water resource management over time (i.e., composite community sensitivity and environmental awareness variables).

It is worth noting that Wei et al.'s (2017) results are not particular to a specific basin, but rather are intended to reflect a broader national or regional view. Validated SH models that endogenized water-related beliefs and norms are distinct from regression-based models that are not causal (e.g., Wei et al., 2017). The in-built nonlinear dynamics allow possible "extrapolation" of the coupled human-water dynamics across a gradient of hydroclimates, societies, and economies, although this requires more work and testing. Similar to regionalization techniques in hydrological modeling, sociohydrological regionalization will consider how the parameters of the coupled SH model, such as the curvature parameter of the distribution function that trades off envirocentric values with anthropocentric values (Roobavannan et al., 2017), vary across different societies. Regression-based models cannot be extrapolated to another place or time as there are no causal linkages provided to explain the transitional shifts in societal values observed therein. In other words, regression models that do not internalize coupled human water system dynamics can at best be used for "interpolation" (i.e., can only explain the dynamics within the domain of the data) or data analysis. Nonetheless, verification of coupled models with data such as those presented in Wei et al. (2017) is important, as it enables the discovery of fundamental principles of human behavior through the validation of internal dynamics within the coupled models and ultimately aids in the generalization of sociohydrologic system dynamics. The following shows how newspaper content analysis effectively plays the same informative role as FSR (i.e., a proxy for condition of ecology) in modeling water-related endogenous behavior.

In order to illustrate how newspaper content analysis serves as a complementary source of information that can be used in sociohydrological modeling, the Wei et al. (2017) 
data was used to recalibrate the "environmental awareness" state variable of van Emmerik et al. (2014).

Instead of wetland storage, which was used in van Emmerik et al. (2014), the FSR $(r)$ is now used as a proxy of environment health. The temporal dynamics of environmental awareness $(E)$ is assumed to be given by the following differential equation:

$$
\frac{\mathrm{d} E}{\mathrm{~d} t}=\varepsilon(r)
$$

where $\varepsilon(r)$ is the rate of accumulation or depletion of environmental awareness, which is a function of $r$. The functional form of $\varepsilon(r)$ is assumed to be given by the following:

$\varepsilon(r)=\left\{\begin{array}{ll}\alpha[\exp (\beta r)-1], & r<r_{\mathrm{c}} \\ -\lambda, & r>r_{\mathrm{c}}\end{array}\right.$,

where $r_{\mathrm{c}}$ is the critical FSR below which environmental awareness is expected to increase exponentially (governed by parameters $\alpha$ and $\beta$ and $\lambda$ ) is the dissipation rate of environmental awareness when the ecosystem is healthy, i.e., $r>r_{\mathrm{c}}$. The FSR, $r$ (Yoshikawa et al., 2014), is estimated by the following power law function:

$r=\beta_{0} Q_{B}^{\beta_{1}}$,

where $Q_{B}$ is the flow in the downstream streamflow (i.e., environmental flow), and $\beta_{0}$ and $\beta_{1}$ are parameters of the FSR index (Yoshikawa et al., 2014).

Values of the parameters $\alpha, \beta, \lambda$, and $r_{\mathrm{c}}$ need to be calibrated. In the absence of social data to calibrate the model, van Emmerik et al. (2014) used other basin-wide hydrological data to calibrate the model. Here we use the Wei et al. (2017) data to calibrate the model parameters, through application of the generalized likelihood uncertainty estimation (GLUE) method. Initial estimates for the parameters are obtained manually to ensure essential dynamics are captured. After that, 100000 random samples of parameters (uniform sampling) that lie within the range of 50 to $150 \%$ of the initial values are obtained.

Figure 5a shows the modeled environmental awareness by van Emmerik et al. (2014) and a comparison with that calibrated to the Wei et al. (2017) data (Fig. 5b). The environmental awareness $(E$, Fig. 5a) bears a remarkable similarity to that obtained by Wei et al. (2017) through newspaper content analysis. Even though van Emmerik et al. (2014) at that time was not privy to the Wei et al. (2017) data, the model already succeeded in capturing the change in community's values and norms regarding water resources. While naturally attracting criticism for the lack of direct calibration, in hindsight the validity of the approach may now be appreciated, and new social data such as Wei et al.'s (2017) can be used to validate predictions of changing values and norms. Figure $5 \mathrm{~b}$ shows how with foresight and with availability of complementary societal values data of Wei et al. (2017) (see dashed line), the FSR can robustly simulate $E$. In doing so it provides independent validation of the model results of van Emmerik et al. (2014) and the approach that was adopted at the time.

\section{From place-based to generalized models: challenges and opportunities}

Community sensitivity and environmental awareness are defined to capture the changing values and norms in different sociohydrological models. In van Emmerik et al. (2014) a simple memory function governed by wetland storage sufficed, whereas in Elshafei et al. (2014) more complex community sensitivity equations were introduced, both linking water-use-related values, beliefs, norms, and behavior through two-way feedbacks. Roobavannan et al. (2017) advanced this a step further by representing community-level belief about the environment, i.e., community sensitivity, as a consequence of the distribution of weights that individuals attach to envirocentric versus anthropocentric values. Such a distribution was made contextual by making it dependent on economic diversification. The endogenous treatment of values and norms by these studies have implicitly followed the general logic of elements of the VBN theory presented above, even if this was originally unintended (see the feedback from actions to beliefs in Fig. 2) and have therefore responded to the challenges of incorporating feedbacks from water use behavior to beliefs and water management norms, consistent with the notion of endogenous and dynamic culture (Caldas et al., 2015).

It should be noted that these variables include values, beliefs, and norms together (Fig. 6). Indeed there is a need to further distinguish and differentiate the variables as they become more reliably observed, which would be realized with progress in SH. A more generalized understanding of community sensitivity can then be developed.

The pathway to generalization of SH models is an important goal that allows future prediction (extrapolation in time) and translation of $\mathrm{SH}$ models at other geographical locations (extrapolation in space). It provides an important means for the adoption of sociohydrology in the practice of longterm or strategic water resource management. Generalization needs to address both the proxies used in $\mathrm{SH}$ modeling and the data used to calibrate them, as recent SH modeling studies have highlighted.

Models provide languages or templates in terms of which the following three aspects can be interpreted: (1) how beliefs and norms depend on values; (2) how values and norms influence individual behavior towards the environment, e.g., the wetland health or releasing environment water for biodiversity; and (3) how proenvironmental behavior of some in the community (e.g., rallies by the Australian green movement) can influence the beliefs of others in the basin and bring about a change in water management (i.e., the feed- 


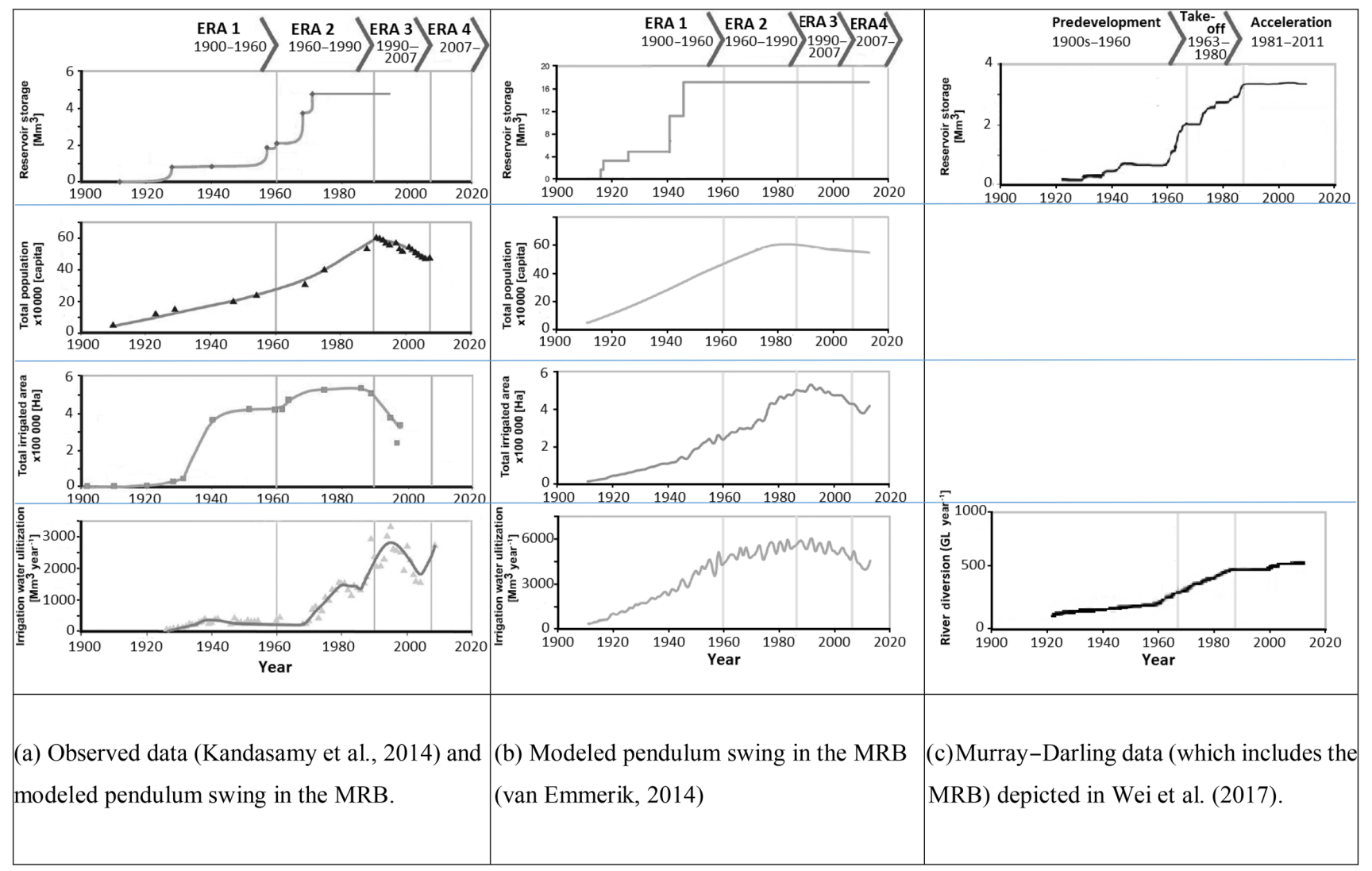

Figure 3. Observed and modeled pendulum swing in the MRB during the period 1910-2013. Era 1 (1900-1980): expansion of agriculture and associated infrastructure; Era 2 (1960-1990): onset of environmental degradation; Era 3 (1990-2007): establishment of widespread environmental degradation; Era 4 (2007-2014): remediation and emergence of environmental customer. The eras correspond to phases in Elshafei et al. (2015): expansion (1911-1960) - aggressive rate of expansion and active modification of water balance; contraction (1960s) - plateau in anthropogenic modification; recession (1970-2002), cumulative negative impacts on economic and environmental well-being; recovery and new equilibrium (2002-present) - adoption of remedial measures; and in Wei et al. (2017): predevelopment (1900s-1960s) societal values dominated by economic development; take-off (1963-1980) - societal values reflected increasing environmental awareness due to outbreak of pollution events; acceleration (1981-2011) - growing shift in societal values towards environmental sustainability.

back). Such templates also enlighten us with variables that need to be measured, so that multiple concepts via the models can be tested and can improve our system understanding.

For example, the policy change in the 1990s in MRB led to increased environmental flow. To interpret this in terms of change in water management norms of the MRB, models need to link beliefs and norms to water use behavior within the basin. This needs information on a range of relevant values such as altruistic values (i.e., healthy MRB for present and future generations, enough money for the next generation) and egoistic values (i.e., making money), along with information on beliefs, norms, and behaviors, such as how water is being used.

\subsection{Measurement of changing norms and values}

Direct measurement of social value is often very difficult, resulting in the use of indirect methods (or proxies). Stud- ies have attempted to understand social values on proenvironmentalism (Bengston 1994; Ives and Kendal, 2013) and could be differentiated based on the method of measurement. Assigned values can be expressed in either monetary or nonmonetary terms, and are relevant to economic and psychology approaches. In a social-science context, assigned values have been quantitatively measured using a variety of techniques, including survey and interview approaches with the help of psychometric scales used in psychology (Bengston, 1994), social experiments in behavioral economics (Janssen et al., 2014; Yu et al., 2016) and content analysis (Seymour et al., 2010; Bark et al., 2016a; Xu and Bengston, 1997; Wei et al., 2017).

Schwartz's framework (Schwartz, 1992) specifies a set of 10 distinct values across cultures, which suggests that these are universal motivations for attitudes and behaviors. $\mathrm{Hu}-$ mans differ mainly in terms of the importance attached to the constellation or structure of these held values. Drawing on 

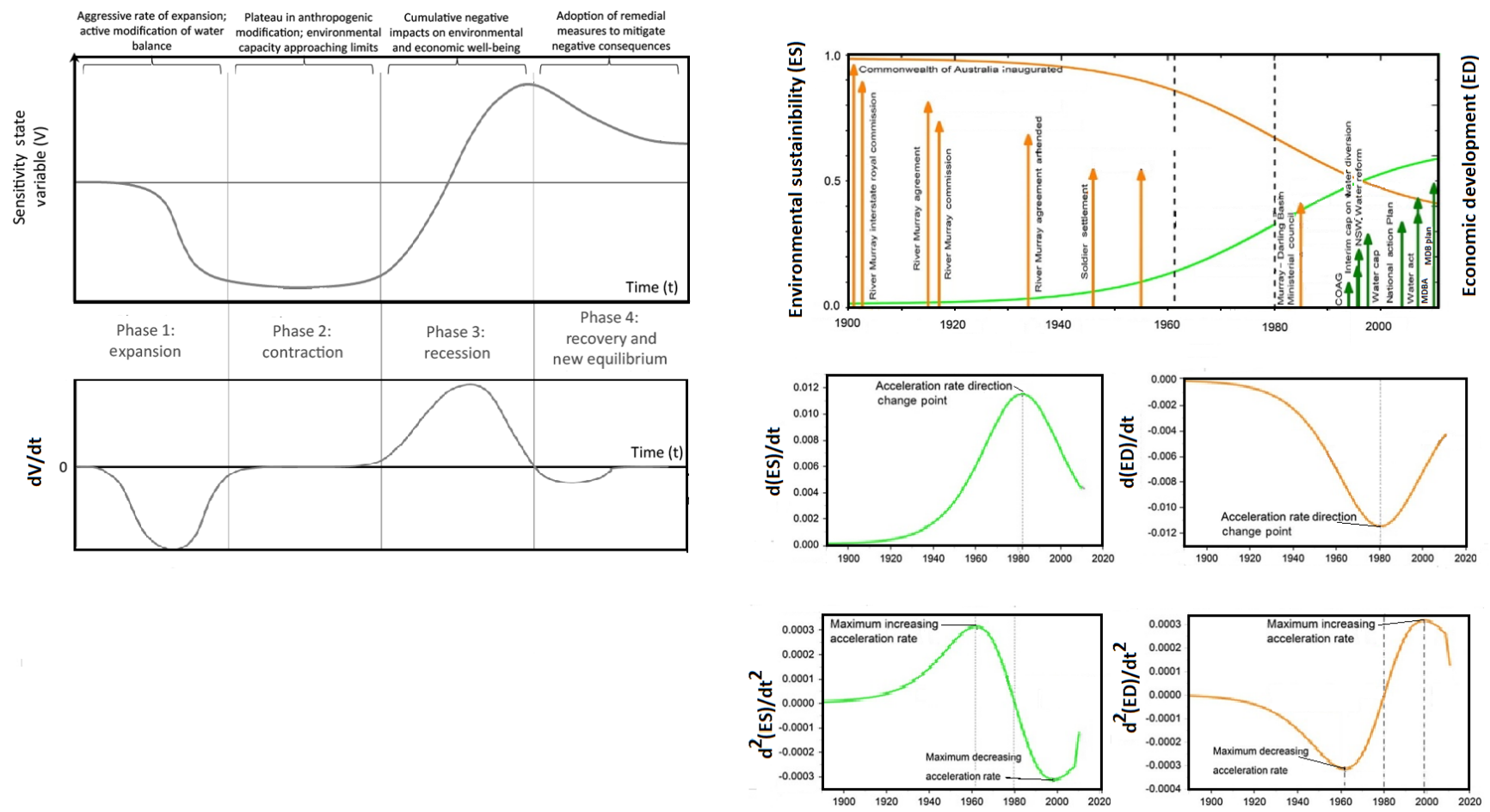

Figure 4. Defining shifts and turning points of stages of societal values.
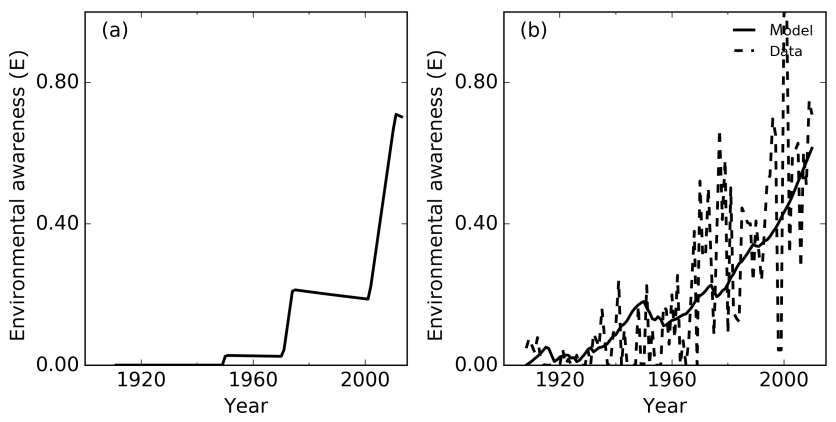

Figure 5. (a) Variation of modeled environmental awareness by van Emmerik et al. (2014) using a model calibrated with hydrological and population data; (b) variation of modeled environmental awareness using a model calibrated (solid line) with societal value data (data from Wei et al., 2017, dashed line) of water resources for environment stability.

Schwartz's framework, values can be measured through survey instruments, which include items that assess the degree to which respondents feel each value is important for their life (Dietz et al., 2005). Following Stern et al. (1999), beliefs (general and specific) and norms - along with Schwartz's value types - have been measured using survey instruments in a wide array of spatiotemporal contexts. These surveybased measures provide cross-sectional indicators. Whether, and how, values, beliefs, and norms are dynamic is an active area of incipient research.

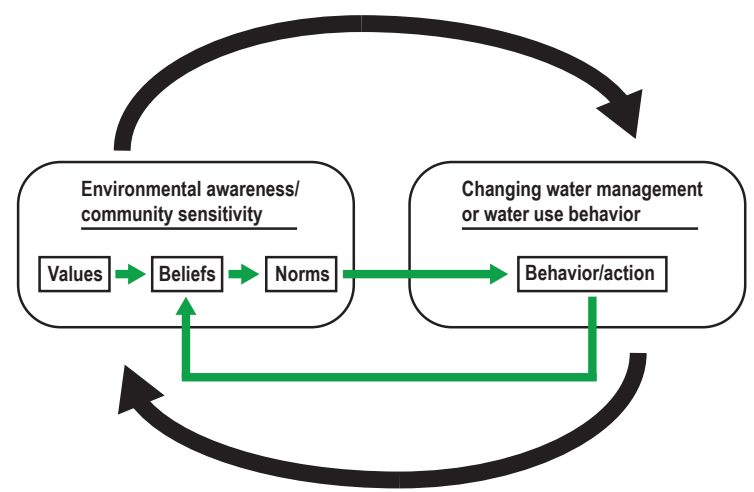

Figure 6. A conceptual diagram of relationships between variables studied in SH modeling and VBN theory. Black arrows show the feedback loops captured in SH modeling and green arrows show relationships that need to be studied in the context of water management.

Economic valuation offers another set of useful approaches to inform natural resource management (Farber et al., 2002; Pande et al., 2011; Loomis et al., 2000; Norton and Noonan, 2007; Wilson and Carpenter, 1999; Bark et al., $2016 b)$. Economic valuation approaches to measuring values are quite distinct from the broader meanings and uses of "values" described above. These approaches include nonmarket valuation (Smith, 1993), contingent valuation (Bateman et al., 2006), and other related techniques, which have been ex- 
tensively used over recent decades and enabled the exploration of how people "trade off" their values in decisionmaking (Freeman, 1993). This enables (i) values to be measured for large and diverse groups of people, (ii) changes in values to be tracked across groups of people or across time, and (iii) models to be developed to predict values based on other factors (e.g., demographics, cultural background). One key limitation of these approaches is that to the extent that values are measured monetarily, these approaches may not accurately capture underlying values to which it is difficult to assign monetary value, but may be more fundamental for decision-making. More generally, there are still unresolved and important questions about value measurement that reflect conceptual and methodological divisions among social sciences and economics. Overcoming these divisions will be crucial for addressing problems in coupled human-water systems.

It is less challenging to observe contemporary waterrelated behavior. However, as the timescale of analysis expands, the task of measuring behavior becomes equally challenging. Paleoclimate proxies such as $\delta^{18} \mathrm{O}$ or tree rings have been extensively used to interpret water availability as well as social organization in the past (Pande and Ertsen, 2014; Staubwasser et al., 2003). These observations can be supplemented by other forms of indirect measurement of waterrelated behavior such as newspaper content analysis, and records of memberships in activist organizations, strengthening proxy observations of proenvironmental behavior in the near past.

\subsection{Utilization of new types of data}

A challenge related to model transferability is generic data needs. If environmental awareness and community sensitivity functions are able to assess some trade-off between envirocentric and anthropocentric values, global socioeconomic data sets such as the World Value Surveys (WVS, 2017) and UN demographic datasets (UN, 2017) might offer the possibility of quantifying values, so that models can be transferred to unmonitored locations. Whether such data sources can be used to quantify such values remains a very important open question.

In the past, the use of soft data in hydrological modeling has been demonstrated to provide additional insights into the functioning of ungauged basins, and has in some cases been used to successfully assess the realism of a model (see e.g., van Emmerik et al., 2015). Similarly, sociohydrological systems face similar problems of extrapolation to other places, as numerical data series do not always exist to calibrate or validate SH models. Wei et al.'s (2017) use of newspaper content data to compute a numerical expression of environmental sustainability and economic development demonstrates the benefits of further exploration of this type of new data sources since it can allow the calibration of SH models, as shown in Fig. 5. Future efforts should therefore not only be limited to developing new SH modeling frameworks, but also entail finding new ways to access information and translate it into numerical expressions, e.g., indices such as FSR, that can be used for model validation and model realism assessment.

A new era of data-driven science (Peters-Lidard et al., 2017) is dawning, with increased computational power, new proxies and alternative data sources. Smart distillation of information from alternative sources (e.g., web databases, social data, other types of big data) may provide the valuable auxiliary data required to take the next step in SH model development and provide an innovative way to find and quantify the social proxies which are currently difficult to justify. This will need to be combined with online data monitoring such as smart sensing and citizen science monitoring as well as field campaigns to validate model results as well as to obtain sociohydrological data relating to, for example, environmental sentiment, local societal values, and land fertility conditions. In the future, sociohydrologists could exploit or mine data/information from such varied sources, leading to the inclusion of big data science in sociohydrology. This new paradigm represents a clear set of opportunities for data-mining and data-driven modeling methods in sociohydrology. These apply machine learning and "computationally intelligent" algorithms to elicit, characterize, quantify, and model the myriad implicit structures and relationships embedded within complex multivariate datasets. In doing so, they offer a pathway for formulating new understandings of the saliency and power of sociohydrologic variables, as well as the interrelationships and behaviors that exist between them (Mount et al., 2016).

\subsection{Comparative sociohydrology studies}

Parameters are used to calibrate the proxies to fit local basin data. Comparative studies from several basins will enable better interpretation of what model parameters mean and their character. For example, Roobavannan et al. (2017)'s model of endogenous behavior could be made more sociohydrologically meaningful. Its attractiveness parameter relates migration to the difference in unemployment within and outside the basin. A more meaningful representation of this variable, for example in terms of the cost of migration, such as moving costs and the cost of obtaining new skills away from water-based employment, will enable regionalization of associated parameter values and the transfer of models from data-intensive basins such as the MRB to data-scarce basins such as the Aral Sea.

Comparative studies can also provide the data to develop regional relationships for SH model parameters. Similar to regionalization techniques in hydrological modeling (Asong et al., 2015; Buytaert and Beven, 2009; Götzinger and Bárdossy, 2007; Merz and Blöschl, 2004; Yadav et al., 2007; Blöschl et al., 2013), sociohydrological regionalization will define how the parameters of the coupled SH model vary with 
different societies and basins. Once defined, regional curves may be used to interpolate parameters and hence models to ungauged locations. Initial efforts have already been made in Elshafei et al. (2016), but these need to be improved and validated through more independent comparative studies. Yet another possibility could be investigation of a Budyko-type curve for coupled human-water systems with endogenous values and norms that will enable extrapolation of emergent behaviors in space and time. Comparative assessment will also put to the test theories such as those that propose values and norms as emergent properties of a coupled humanwater system, such that all its biological constituents, including humans and vegetation, obey certain metabolic scaling laws (Fischer-Kowalski, 1998; Silva et al., 2006).

In this regard, a new working group on comparative sociohydrology within Panta Rhei has been launched to serve this purpose (Fuqiang Tian, personal communication). It plans to obtain sociohydrological data from diverse river basins such as Tarim in China, Murrumbidgee in Australia, and Kissimmee in USA, including historical documentation of the evolution of coupled human-water systems to develop a generalized understanding of coupled human-water behavior. This is being done through comparative analysis to identify and interpret diverse emergent behavior such as farmer suicides in less developed and developed countries such as India and Australia, respectively; "pendulum swing" in basins in China, USA, and Australia; and the levee effect versus memory effect in flood plains across the globe. Such comparative analyses can prove to be very constructive in identifying general principles that govern dynamic changes in values and norms.

\section{Conclusions}

Recent sociohydrological studies in Australia have moved closer toward integration with key social-science theories of perception and behavior and have taken a key step toward endogenizing values and norms. These models are internally consistent with patterns observed with proxy data of environmental awareness and water policy change, such as the newspaper-article-based proxies of Wei et al. (2017). However, such theoretically and empirically consistent models are only the beginning of the way forward to generalizing models and their predictions for sustainable water management.

Human culture - comprised of values, beliefs, and norms - is key to understanding stability and change in coupled human-water systems. Often, such variables and related closure relationships within sociohydrological models are latent and hard to observe. This poses challenges in testing and confirming the realism of assumed relationships. However, with the advent of the information-intense era, diverse proxy data sources such as citizen science observatories and social media can be harnessed and novel big data algorithms can be used to process them in a form that can be of use to sociohydrological models.

Yet such opportunities can only build confidence in our place-based understanding of a sociohydrological phenomenon such as the pendulum swing observed in the Murrumbidgee river basin. What we need are generalized relationships or principles underlying emergent phenomena if we are to stand up to the challenge of making predictions in ungauged locations in space and time. This clearly calls for more place-based studies, both past and present and across spatiotemporal scales, that are backed up by novel sociohydrological observations such as historical accounts and sociocentric data. A comparative analysis of such studies in which similar emergent phenomena have been observed would help synthesize the underlying sociohydrological principles.

Data availability. The model data used in this study are deposited at https://doi.org/10.6084/m9.figshare.5881393.v1.

Competing interests. The authors declare that they have no conflict of interest.

Acknowledgements. This paper falls within the framework of the Panta-Rhei Research Initiative of the International Association of Hydrological Sciences (IAHS). We would like to acknowledge the US National Science Foundation's Socio-Environmental Synthesis Center (SESYNC; NSF award DBI-1052875) for their support of the project "Toward Socio-hydrologic Synthesis: Modeling the Co-evolutionary Dynamics of Coupled Human, Water, and Ecological System". The paper benefited from constructive criticism and suggestions from reviewers Yongping Wei and Xi Chen and editor Anas Ghadouani for which we are grateful.

Edited by: Anas Ghadouani

Reviewed by: Yongping Wei and Xi Chen

\section{References}

Andreoni, J.: Giving with impure altruism: Applications to charity and ricardian equivalence, J. Polit. Econ., 97, 1447-1458, 1989.

Asong, Z. E., Khaliq, M. N., and Wheater, H. S.: Regionalization of precipitation characteristics in the Canadian Prairie Provinces using large-scale atmospheric covariates and geophysical attributes, Stoch Environ. Res. Risk A., 29, 875-892, 2015.

Bai, X., van der Leeuw, S., O’Brien, K., Berkhout, F., Biermann, F., Brondizio, E. S., Cudennec, C., Dearing, J., Duraiappah, A., Glaser, M., Revkin, A., Steffen, W., and Syvitski, J.: Plausible and desirable futures in the Anthropocene: A new research agenda, Global Environ. Change, 39, 351-362, 2016.

Banerjee, A. V. and Newman, A. F.: Occupational choice and the process of development, J. Polit. Econ., 101, 274-298, 1993. 
Bark, R. H., Robinson, C. J., and Flessa, K. W.: Tracking cultural ecosystem services: Water chasing the Colorado River restoration pulse flow, Ecol. Econ., 127, 165-172, 2016a.

Bark, R. H., Colloff, M. J., Hatton MacDonald, D., Pollino, C. A., Jackson, S., and Crossman, N. D.: Integrated valuation of ecosystem services obtained from restoring water to the environment in a major regulated river basin. Ecosyst. Serv., 22, 381-391, 2016 b.

Bateman, I. J., Cole, M. A., Georgiou, S., and Hadley, D. J.: Comparing contingent valuation and contingent ranking: A case study considering the benefits of urban river water quality improvements, J. Environ. Manage., 79, 221-231, 2006.

Bengston, D. N.: Changing forest values and ecosystem management, Soc. Nat. Resour., 7, 515-533, 1994.

Blair, P. and Buytaert, W.: Socio-hydrological modelling: a review asking "why, what and how?", Hydrol. Earth Syst. Sci., 20, 443478, https://doi.org/10.5194/hess-20-443-2016, 2016.

Blöschl, G., Sivapalan, M., Wagener, T., Viglione, A., and Savenije, H. H. G.: Runoff Prediction in Ungauged Basins - Synthesis across Processes, Places and Scales, Cambridge University Press, Cambridge, UK, p. 500, 2013.

Buytaert, W. and Beven, K.: Regionalization as a learning process, Water Resour. Res., 45, 1-13, 2009.

Caldas, M. M., Sanderson, M. R., Mather, M., Daniels, M. D., Bergtold, J. S., Aistrup, J., Heier Stamm, J. L., Haukos, D., Douglas-Mankin, K., Sheshukov, A. Y., Lopez-Carr, D.: Opinion: Endogenizing culture in sustainability science research and policy, P. Natl. Acad. Sci. USA, 112, 8157-8159, https://doi.org/10.1073/pnas.1510010112, 2015.

Chen, X., Wang, D., Tian, F., and Sivapalan, M.: From Channelization to Restoration: Sociohydrologic Modeling with Changing Community Preferences in the Kissimmee River Basin, Florida, Water Resour. Res., 52, 1227-1244, https://doi.org/10.1002/2015WR018194, 2016.

Di Baldassarre, G., Viglione, A., Carr, G., Kuil, L., Salinas, J. L., and Blöschl, G.: Socio-hydrology: conceptualising humanflood interactions, Hydrol. Earth Syst. Sci., 17, 3295-3303, https://doi.org/10.5194/hess-17-3295-2013, 2013.

Dietz, T.: Environmental values, in: Oxford Handbook of Values, edited by: Brosch, T. and Sander, D., Oxford University Press, Oxford, UK, 329-349, 2015.

Dietz, T., Fitzgerald, A., and Shwom, R.: Environmental Values, Annu. Rev. Environ. Resour., 30, 335-372, 2005.

Elshafei, Y., Sivapalan, M., Tonts, M., and Hipsey, M. R.: A prototype framework for models of socio-hydrology: identification of key feedback loops and parameterisation approach, Hydrol. Earth Syst. Sci., 18, 2141-2166, https://doi.org/10.5194/hess-182141-2014, 2014.

Elshafei, Y., Coletti, J. Z., Sivapalan, M., and Hipsey, M. R.: A model of the socio-hydrologic dynamics in a semiarid catchment: Isolating feedbacks in the coupled human-hydrology system, Water Resour. Res., 51, 6442-6471, 2015.

Elshafei, Y., Tonts, M., Sivapalan, M., and Hipsey, M. R.: Sensitivity of emergent sociohydrologic dynamics to internal system properties and external sociopolitical factors: Implications for water management, Water Resour. Res., 52, 4944-4966, 2016.

Falkenmark, M. and Rockström, J.: Balancing Water for Humans and Nature, Earthscan, New York, USA, 247 pp., 2004.
Farber, S. C., Costanza, R., and Wilson, M. A.: Economic and ecological concepts for valuing ecosystem services, Ecol. Econ., 41, 375-392, 2002.

Fischer-Kowalski, M.: Society's metabolism. The intellectual histroy of materials flow analysis, Part I, 1860-1970, J. Ind. Ecol., 2, 61-136, 1998.

Freeman, A. M.: The Measurement of Environmental and Resource Values: Theory and Methods, Resources for the Future, Washington, D.C., USA, 1-459, 1993.

Garcia, M., Portney, K., and Islam, S.: A question driven sociohydrological modeling process, Hydrol. Earth Syst. Sci., 20, 73 92, https://doi.org/10.5194/hess-20-73-2016, 2016.

Götzinger, J. and Bárdossy, A.: Comparison of four regionalisation methods for a distributed hydrological model, J. Hydrol., 333, 374-384, 2007.

Ives, C. D. and Kendal, D.: Values and attitudes of the urban public towards peri-urban agricultural land, Land Use Policy, 34, 80-90, 2013.

Ives, C. D. and Kendal, D.: The role of social values in the management of ecological systems, J. Environ. Manage., 144, 67-72, 2014.

Janssen, M. A., Lee, A., and Waring, T. M.: Experimental platforms for behavioral experiments on social-ecological systems, Ecol. Soc., 19, 20, 2014.

Kandasamy, J., Sounthararajah, D., Sivabalan, P., Chanan, A., Vigneswaran, S., and Sivapalan, M.: Socio-hydrologic drivers of the pendulum swing between agricultural development and environmental health: a case study from Murrumbidgee River basin, Australia, Hydrol. Earth Syst. Sci., 18, 1027-1041, https://doi.org/10.5194/hess-18-1027-2014, 2014.

Liu, Y., Tian, F., Hu, H., and Sivapalan, M.: Socio-hydrologic perspectives of the co-evolution of humans and water in the Tarim River basin, Western China: the Taiji-Tire model, Hydrol. Earth Syst. Sci., 18, 1289-1303, https://doi.org/10.5194/hess-18-12892014, 2014.

Loomis, J., Kent, P., Strange, L., Fausch, K., and Covich, A.: Measuring the total economic value of restoring ecosystem services in an impaired river basin: Results from a contingent valuation survey, Ecol. Econ., 33, 103-117, 2000.

Merz, R. and Blöschl, G.: Regionalisation of catchment model parameters, J. Hydrol., 287, 95-123, 2004.

Millennium Ecosystem Assessment: Ecosystems and Human Wellbeing: Current State and Trends, Vol. 1, Island Press, Washington, D.C., USA, 2005.

Mount, N. J., Maier, H. R., Toth, E., Elshorbagy, A., Solomatine, D., Chang, F.-J., and Abrahart, R. J.: Data-driven modelling approaches for socio-hydrology: Opportunities and challenges within the Panta Rhei Science Plan, Hydrolog. Sci. J., 61, 11921208, 2016.

Norton, B., Costanza, R., and Bishop, R. C.: The evolution of preferences why "sovereign" preferences may not lead to sustainable policies and what to do about it, Ecol. Econ., 24, 193-211, 1998.

Norton, B. G. and Noonan, D.: Ecology and valuation: Big changes needed, Ecol. Econ., 63, 664-675, 2007.

Pande, S. and Ertsen, M.: Endogenous change: on cooperation and water availability in two ancient societies, Hydrol. Earth Syst. Sci., 18, 1745-1760, https://doi.org/10.5194/hess-18-1745-2014, 2014. 
Pande, S. and Savenije, H. H. G.: A sociohydrological model for smallholder farmers in Maharashtra, India, Water Resour. Res., 52, 1923-1947, 2016.

Pande, S. and Sivapalan, M.: Progress in socio-hydrology: a metaanalysis of challenges and opportunities, Wiley Interdiscip. Rev. Water, 4, 1-18, 2016.

Pande, S., van Den Boom, B., Savenije, H. H. G., and Gosain, A. K.: Water valuation at basin scale with application to western India, Ecol. Econ., 70, 2416-2428, 2011.

Peters-Lidard, C. D., Clark, M., Samaniego, L., Verhoest, N. E. C., van Emmerik, T., Uijlenhoet, R., Achieng, K., Franz, T. E., and Woods, R.: Scaling, similarity, and the fourth paradigm for hydrology, Hydrol. Earth Syst. Sci., 21, 3701-3713, https://doi.org/10.5194/hess-21-3701-2017, 2017.

Rijsberman, F. R.: Water scarcity: Fact or fiction?, Agr. Water Manage., 80, 5-22, 2006.

Roobavannan, M., Kandasamy, J., Pande, S., Vigneswaran, S., and Sivapalan, M.: Role of sectoral transformation in evolution of the water management in agricultural catchments: A socio-hydrologic analysis, Water Resour. Res., 53, 8344-8365, https://doi.org/10.1002/2017WR020671, 2017.

Schwartz, S. H.: Universals in the content and structure of values: Theory and empirical tests in 20 countries, edited by: Zanna, M., Adv. Exp. Soc. Psychol., 25, 1-65, 1992.

Schwartz, S. H., Melech, G., Lehmann, A., Burgess, S., Harris, M., and Owens, V.: Extending the Cross-Cultural Validity of the Theory of Basic Human Values with a Different Method of Measurement, J. Cross. Cult. Psychol., 32, 519-542, 2001.

Seymour, E., Curtis, A., Pannell, D., Allan, C., and Roberts, A.: Understanding the role of assigned values in natural resource management, Aust. J. Environ. Manage., 17, 142-153, 2010.

Silva, J. K. L., Garcia, G. J. M., and Barbosa, L. A.: Allometric scaling laws of metabolism, Phys. Life Rev., 3, 229-261, 2006.

Sivapalan, M. and Blöschl, G.: Time scale interactions and the coevolution of humans and water, Water Resour. Res., 51, 69887022, 2015.

Sivapalan, M., Konar, M., Srinivasan, V., Chhatre, A., Wutich, A., Scott, C. A., and Wescoat, J. L.: Socio-hydrology: Use-inspired water sustainability science for the Anthropocene, Earth's Future, 2, 225-230, 2014.

Smith, V. K.: Nonmarket valuation of environmental resources: An interpretive appraisal, Land Econ., 69, 1-26, 1993.

Srinivasan, V., Sanderson, M., Garcia, M., Konar, M., Blöschl, G., and Sivapalan, M.: Prediction in a sociohydrological world, Hydrolog. Sci. J., 62, 338-345, https://doi.org/10.1080/02626667.2016.1253844, 2016.

Srinivasan, V., Konar, M., and Sivapalan, M.: A dynamic framework for water security, in: Water Security, Elsevier, 1, 12-20, https://doi.org/10.1016/j.wasec.2017.03.001, 2017.

Staubwasser, M., Sirocko, F., Grootes, P. M., and Segl, M.: Climate change at the $4.2 \mathrm{ka}$ BP termination of the Indus valley civilization and Holocene south Asian monsoon variability, Geophys. Res. Lett., 30, 3-6, 2003.
Stern, P. C.: Toward a coherent theory of environmentally significant behavior, J. Soc. Issues, 56, 407-424, 2000.

Stern, P. C., Dietz, T., Abel, T., Guagnano, G. A., and Kalof, L.: A value-belief-norm theory of support for social movements: the case of environmentalism, Human Ecol. Rev., 6, 81-98, 1999.

UN - United Nations: UN-Population Division, available at: http://www.un.org/en/development/desa/population/ publications/dataset/, last access: 18 July 2017.

van Emmerik, T., Mulder, G., Eilander, D., Piet, M., and Savenije, H. H. G.: Predicting the ungauged basin: Model validation and realism assessment, Front. Earth Sci., 3, 62, https://doi.org/10.3389/feart.2015.00062, 2015.

van Emmerik, T. H. M., Li, Z., Sivapalan, M., Pande, S., Kandasamy, J., Savenije, H. H. G., Chanan, A., and Vigneswaran, S.: Socio-hydrologic modeling to understand and mediate the competition for water between agriculture development and environmental health: Murrumbidgee River basin, Australia, Hydrol. Earth Syst. Sci., 18, 4239-4259, https://doi.org/10.5194/hess-184239-2014, 2014.

Wei, J., Wei, Y., and Western, A.: Evolution of the societal value of water resources for economic development versus environmental sustainability in Australia from 1843 to 2011, Global Environ. Change, 42, 82-92, 2017.

Wescoat Jr., J. L.: Reconstructing the duty of water: a study of emergent norms in socio-hydrology, Hydrol. Earth Syst. Sci., 17, 4759-4768, https://doi.org/10.5194/hess-17-4759-2013, 2013.

Wilson, M. A. and Carpenter, S. R.: Economic valuation of freshwater ecosystem services in the United States: 1971-1997, Ecol. Appl., 9, 772-783, 1999.

WVS - World Values Survey: World Values Survey-Wave 7, available at: http://www.worldvaluessurvey.org/wvs.jsp, last access: 18 July 2017.

Xiong, Y., Wei, Y., Zhang, Z., and Wei, J.: Evolution of China's water issue framed in Chinese mainstream media, Ambio, 45, 241-251, https://doi.org/10.1007/s13280-015-0716-y, 2016.

$\mathrm{Xu}, \mathrm{Z}$. and Bengston, D. N.: Trends in national forest values among forestry professionals, environmentalists, and the news media, 1982-1993, Soc. Nat. Resour., 10, 43-59, 1997.

Yadav, M., Wagener, T., and Gupta, H. V.: Regionalization of constraints on expected watershed response for improved predictions in ungauged basins, Adv. Water Resour., 30, 1756-1774, https://doi.org/10.1016/j.advwatres.2007.01.005, 2007.

Yoshikawa, S., Yanagawa, A., Iwasaki, Y., Sui, P., Koirala, S., Hirano, K., Khajuria, A., Mahendran, R., Hirabayashi, Y., Yoshimura, C., and Kanae, S.: Illustrating a new global-scale approach to estimating potential reduction in fish species richness due to flow alteration, Hydrol. Earth Syst. Sci., 18, 621-630, https://doi.org/10.5194/hess-18-621-2014, 2014.

Yu, D. J., Shin, H. C., Pérez, I., Anderies, J. M., and Janssen, M. A.: Learning for resilience-based management: Generating hypotheses from a behavioral study, Global Environ. Change, 37, 69-78, 2016 . 